\title{
Pediatric endocrinologists' practices in screening for celiac disease in patients with type 1 diabetes
}

\author{
Liana Gabriel ${ }^{2{ }^{+*}}$, Michael J. Pettei ${ }^{1 \dagger}$, Dennis E. Carey ${ }^{1 \dagger}$, Graeme R. Frank $^{1 \dagger}$, Paula M. Kreitzer ${ }^{1 \dagger}$ and Phyllis W. Speiser ${ }^{1 \dagger}$
}

*Correspondence: ligabrie@montefiore.org

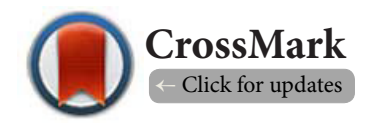

'These authors contributed equally to this work.

'The Divisions of Pediatric Endocrinology \& Gastroenterology (MP), The Steven \& Alexandra Cohen Children's Medical Center of NY, 269-01 76th Avenue, New Hyde Park, NY, 11040, USA.

${ }^{2}$ The Division of Pediatric Endocrinology, Winthrop University Hospital, 259 1st St Mineola, NY, 11501, USA.

\begin{abstract}
Background: Undiagnosed CD may lead to unstable blood glucose levels, weight loss, growth failure, osteopenia. The primary objective of the study was to review CD screening practices in one center, describe symptoms at the time of diagnosis, and implement screening guidelines. The secondary objective was to carry out an educational intervention, to review practices after screening guidelines were established.

Methods: A total of 532 charts of patients with T1D treated in our center from 2007-2009 were reviewed. Data collected included times of screenings for CD, results of serologic tests, symptoms, and intestinal biopsy results. Two hundred and fifty charts from 2010-2012 were reviewed after an educational intervention with the same group of physicians.

Results: After the educational intervention to encourage implementation of screening guidelines, there was a statistically significant reduction in the interval between the diagnosis of T1D and first screening for CD. The majority were diagnosed in the first year after T1D diagnosis, only 30\% were symptomatic.

Conclusion: Our results suggest that patients with T1D should be screened within the first several months after diagnosis, on an annual basis thereafter, even in the absence of gastroenterologic symptoms of CD. This will allow early dietary intervention and reduce morbidities in this high-risk population.
\end{abstract}

Keywords: Diabetes mellitus, type 1, celiac disease, child

\section{Introduction}

Celiac disease (CD) and type 1 diabetes (T1D) are distinct autoimmune disorders that share a common genetic origin [1]. It is one of the most frequent autoimmune disorders occurring in T1D. The prevalence of CD in T1D ranges from 3-16\%, with a mean approximately $8 \%$ worldwide. The highest occurrence has been reported in a study performed in Algeria with a celiac prevalence of $16.4 \%$ in type 1 diabetes patients [2]. The overall prevalence in the general population is only $1 \%$ [3].

Currently, there are no uniform screening guidelines for celiac disease in the T1D population. The gastroenterological association, NASPGHN (North American Society of Pediatric Gastroenterology Hepatology and Nutrition) recommends serologic screening for patients at high risk, including children with type 1 DM after 3 years of age [4]. Conversely, the American
Gastroenterological Association guidelines do not advocate screening such persons if they are truly asymptomatic, but recommends that physicians consider screening in the event patients develop any clinical manifestations associated with $C D$ [5]. Recent guidelines from the American College of Gastroenterology also demonstrate a reluctance to recommend testing asymptomatic individuals [3]. Contrarily, ISPAD (International Society for Pediatric and Adolescent Diabetes) recommends screening for $C D$ at the time of T1D diagnosis, annually for the first five years and every second year thereafter [6].

The American Diabetes Association also recommends screening soon after diagnosis and to consider testing in symptomatic patients. This recommendation is based on level E evidence (Expert consensus or clinical experience) [7]. However, there is a need for better clinical evidence that justifies such screen- 
ing. Pediatric endocrinologists need to be cognizant of the fact that clinical manifestations of $C D$ are variable and often subtle. A Greek study reported that in their cohort of Type 1 diabetes patients most manifested mild growth impairment or no symptoms at all [8]. Narula et al., found that in their population, although the majority of T1D patients with CD were asymptomatic, many reported subtle gastrointestinal complaints, most commonly flatulence [9].

Individuals with T1D and undiagnosed CD may experience weight loss, growth failure, osteopenia and unstable blood glucose levels. Specifically, there is an increased risk for symptomatic hypoglycemia among T1D patients in the 6 months after diagnosis of celiac disease [10]. A Danish study demonstrated that patients with celiac disease who were placed on a gluten-free diet showed an improvement in height, weight standard deviation score, hemoglobin and ferritin levels at their 2 year follow up [11]. The most serious, although rare, long term morbidity is gastrointestinal malignancy [12].

Data from a recent study suggest that TTG-A by ELISA along with a quantitative measure of serum IgA levels (to evaluate for $\lg$ A deficiency) remains a superior serologic marker for screening [13]. Brusca et al., highlighted in their study that the combination of TTG-A and deamidated antigliadin antibody increases the accuracy of $C D$ diagnosis in very young children with sensitivity increasing from $77.9 \%$ with TTG alone to 84.2 $\%$ when combined with deamidated antigliadin antibody, maintaining a specificity of $100 \%$ [14]. HLA typing can be used to identify whether a patient carries the haplotype DQ2 or DQ8 that is necessary for CD to develop. The absence of DQ2 and DQ8 essentially excludes the diagnosis of celiac disease [3]. In fact, the European Society for Pediatric Gastroenterology, Hepatology and Nutrition (ESPGHAN) recommends HLA testing in patients with T1D as the initial step in screening patients with T1D. If HLA testing is negative, no further testing is recommended. If a patient is DQ8 and/or DQ2 positive, homozygous for only the $\beta$-chains of the HLA-DQ2 complex (DQB1*0202), or HLA testing is not done, then a TTG-A and total IgA determination should be performed, based on their guidelines [15]. However, duodenal biopsy is still definitive.

The primary objective of the study was to review CD screening practices in one center, describe symptoms at the time of diagnosis, and implement screening guidelines. The secondary objective was to implement an educational intervention and to review practices after screening guidelines were established.

\section{Materials and methods}

In the first part of the study, five hundred and thirty two (532) consecutive charts were reviewed from patients with Type 1 diabetes treated in the Pediatric Endocrinology outpatient center at Cohen Children's Medical Center of NY from 20072009. Those included all new and established T1D patients seen in that time period. The study was approved by the local Institutional Review Board. We collected data related to initial and repeat screenings for $C D$, its frequency, expressed symptoms, and biopsy results.

After the first part of the study was completed, we instituted guidelines for screening T1D patients for celiac disease: Initial CD Screen to be done within the first 3 months of the diagnosis of T1D:

- For patients $>4$ yrs. old, TTG-A (tissue transglutaminase $\lg A$ ) level and serum IgA level;

- For <4 yrs. old, a TTG-A (tissue transglutaminase lgA) level, an AGA-A (antigliadin IgA) level, and quantitative serum IgA level.

Antigliadin antibody (specifically the deamidated form when commercially available) was added as a screening test to the younger group, since TTG-A is not sufficiently sensitive or specific in very young children [14].

All celiac antibody levels were obtained in one commercial laboratory (NSLIJ Laboratories) by ELISA. Levels less than 20 $\mathrm{U} / \mathrm{ml}$ were considered negative, $20-30 \mathrm{U} / \mathrm{ml}$ weakly positive, and greater than $30 \mathrm{U} / \mathrm{ml}$ strongly positive. All patients with a strongly positive celiac antibody, or who had a second weakly positive celiac antibody were recommended for biopsy. IgA deficient individuals had available IgG antibodies (AGA and TTG) and in some cases HLA DQ 2 or DQ 8 analysis (from Prometheus Laboratories, California). Patients were referred to pediatric gastroenterology if TTG-A was positive (or equivocal), or AGA-A was positive, (or equivocal), or if the serum IgA level was low.

Diagnosis of $C D$ was established by standard clinical criteria with the use of endoscopically-obtained small bowel biopsies. All biopsies were obtained at upper endoscopy with propofol anesthesia. At least 4 mucosal biopsies each were obtained from the distal duodenum and duodenal bulb. All specimens were examined by a pediatric pathologist according to the classification of Marsh [16]. A type 1 lesion had normal mucosal architecture but with increased intraepithelial lymphocytes, IEL's. A type 2 lesion had increased IEL's plus elongated crypts, and a type 3 lesion had increased IEL's, elongated crypts and varying degrees of villous atrophy. Celiac disease was diagnosed for type 2 and type 3 lesions. Patients who refused recommended biopsy continued on a regular gluten-containing diet and were eligible for repeat celiac antibody screening at diabetes follow-up. None subsequently agreed to biopsy. If initial screen was negative, then repeat screen yearly for the first 2-3 years, and then periodically (every 2-3 years), with re-screenings until about 10 years post-T1DM diagnosis, or sooner if symptomatic.

After the educational intervention to encourage implementation of screening guidelines, we reviewed another two hundred and fifty (250) consecutive charts of patients with Type 1 diabetes treated in the Pediatric Endocrinology outpatient center at Cohen Children's Medical Center of NY from the mid2010 through mid- 2012. Those two hundred and fifty patients were all new and established T1D patients seen in our center during this time period, excluding children accounted for in 
the first cohort. We again collected data related to initial and repeat screenings for $C D$, its frequency, expressed symptoms, and biopsy results. Descriptive statistics were tabulated and analyzed. Using the method of Kaplan-Meier, time to initial screening and to celiac diagnosis were compared between the two cohorts (Figures 2 and $\mathbf{3}$ ). However, since the first cohort was followed for a longer time period, to make the timing and $C D$ prevalence comparisons more valid, only patients who were newly diagnosed with T1D in the 2 year time periods of 2007-2009 and 2010-2012 were selected for the purposes of statistical analysis. There were 199 newly diagnosed T1D patients in the 2007-2009 cohort, and 235 newly diagnosed patients in the 2010-2012 cohort.

\section{Results}

The entire 2007-2009 cohort included patients 2-23 years of age. Mean age was $14.2 \pm 4.6$ years. Patient demographic data for the first cohort is presented in Table 1. The 2010-2012 cohort included children 2-20 years of age. Mean age was slightly younger at $12.6 \pm 3.8$ years (Table 1 ). Since the entire first cohort was followed for a longer time period, the duration of their diabetes at the time they were first screened, was longer than in the 2010-2012 cohort.

Of the total 532 patients in the initial cohort, 493 (92.7\%) were screened for celiac disease. Of these 493, 25 patients (5.1\%) were serologically positive (Figure 1 ) and were referred to gastroenterology for further evaluation. Fifteen underwent intestinal biopsy for celiac disease, of whom 11 (44\%) were biopsy positive. Only three of those eleven biopsy-proven $C D$ patients $(27 \%)$ reported symptoms. It is important to note, that of those 25 serologically positive on the first screen, 10 refused to undergo the intestinal biopsy.

None of these individuals had symptoms of abdominal pain, bloating, diarrhea, or any other obvious gastrointestinal symptoms. Rather, the only sign reported was an increased incidence of objectively measured hypoglycemic episodes.

Of those 468 patients who had a negative first screen, repeat serologic testing was done in 261 patients (Figure 1, 55.7\%). The average time until repeat celiac screening in the 20072009 cohort was $1.76 \pm 1.04$ yrs. Among these 261 patients, only $5.4 \%$ (14 patients) had positive serology on the second

Table 1. Patient demographics (2007-2009 and 2010-2012 cohorts).

\begin{tabular}{llll}
\hline Demographics item & & 2007-2009 & 2010-2012 \\
\hline Age & Range & $2-23$ yrs & $2-20$ yrs \\
& Mean & $14.2 \pm 4.6$ & $12.6 \pm 3.8$ \\
\hline Age at T1D onset & Range & $0.8-18$ yrs & $1-20.4$ yrs \\
& Mean & $8 \pm 4.2$ & $9.8 \pm 4.1$ \\
\hline Gender & Female & $49.0 \%$ & $46.0 \%$ \\
& Male & $51.0 \%$ & $54.0 \%$ \\
\hline T1D duration & Range & $0-16$ yrs & $0-7$ yrs \\
& Mean & $3 \pm 3.4$ & $0.48 \pm 0.6$ \\
\hline
\end{tabular}

${ }^{\star}$ Duration of T1D at the time of first screen

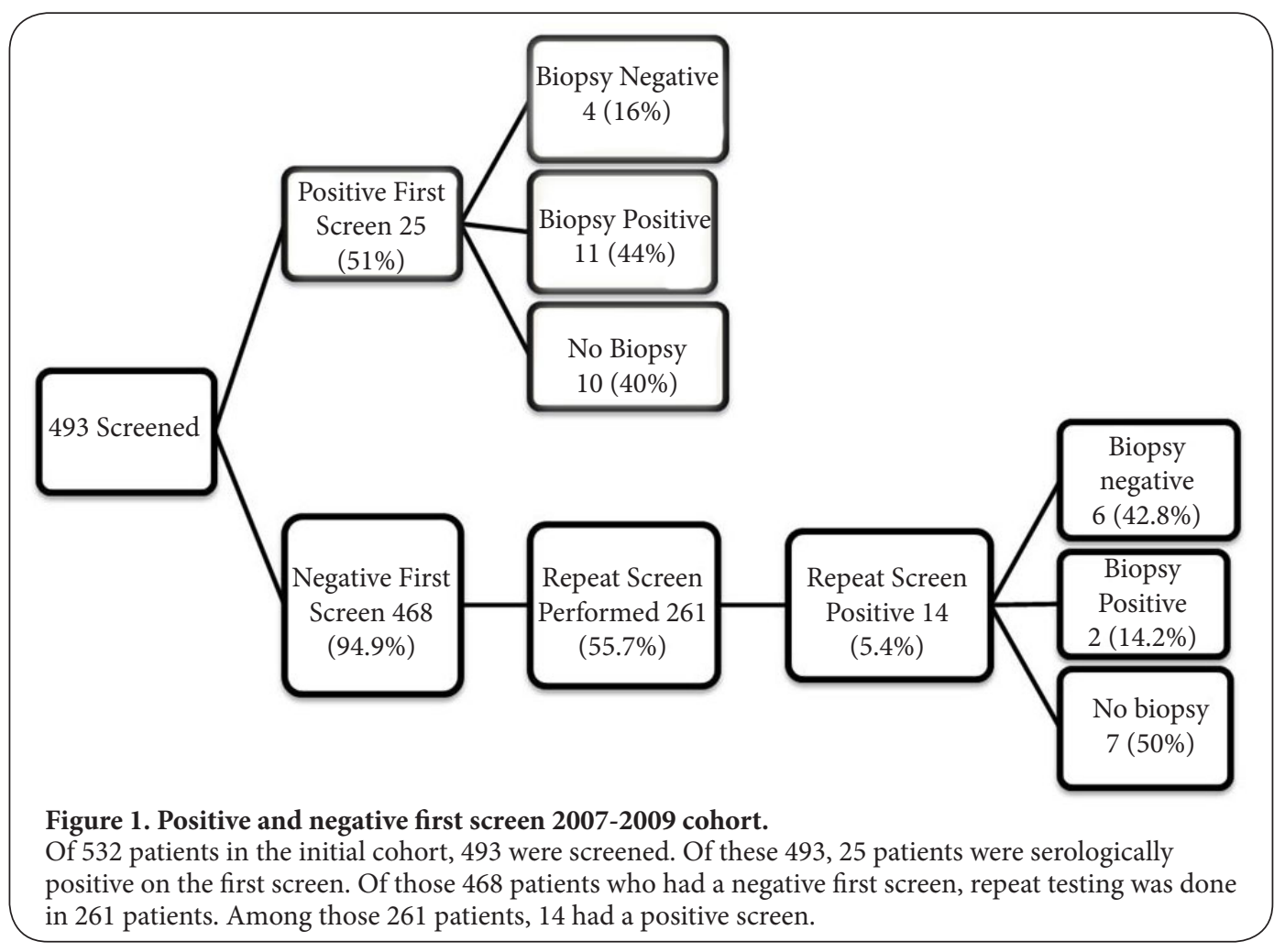




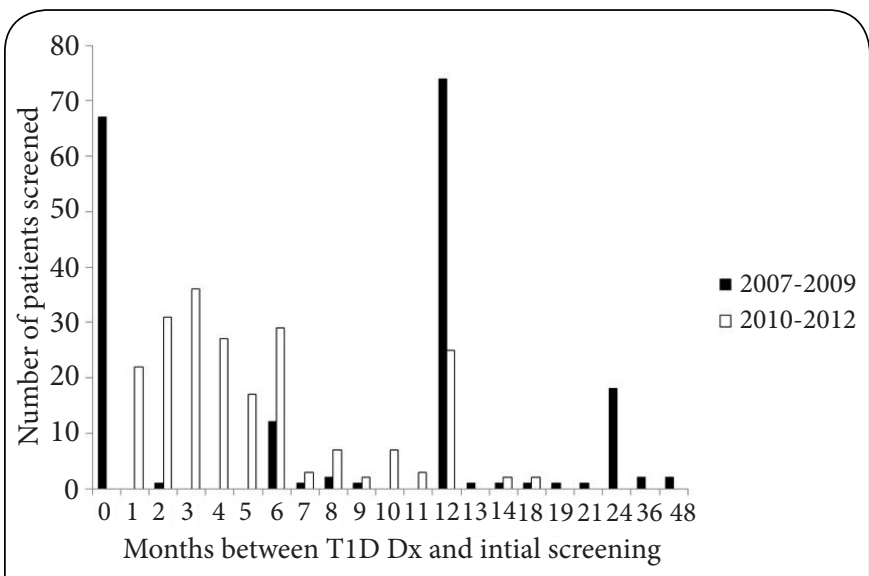

Figure 2. Timing of initial screening, 2007-2009 and 20102012 cohorts.

Figures 2 and 3 demonstrate timing of initial screening and diagnosis of CD only in the subsets of patients from each cohort, diagnosed with T1D in the two time periods. Of those 185 patients in the $2007-2009$ cohort, 83 patients (45\%) were screened in the first half year, contrasted with 149 of those 213 (70\%) who were screened in the same time period in the 20102012 cohort.

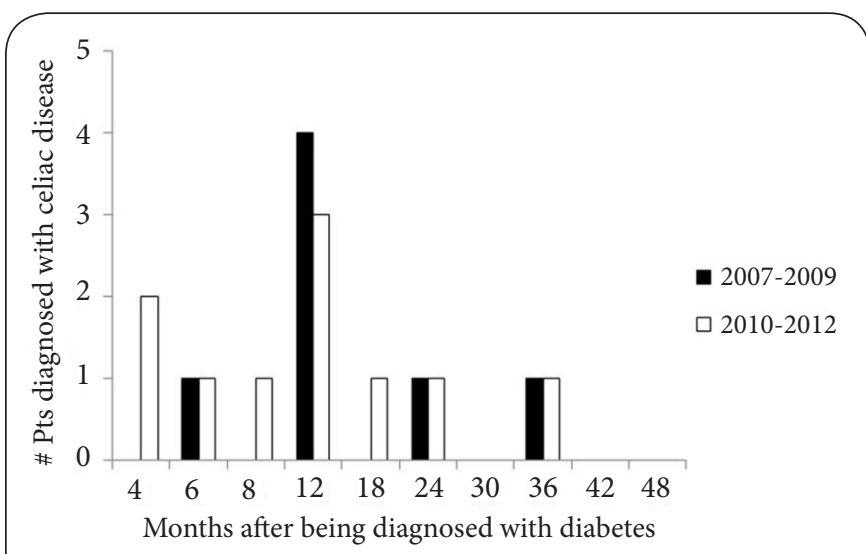

Figure 3. Timing of celiac diagnosis, 2007-2009 and 20102012 cohorts.

About 20\% (2 of 10) of biopsy proven CD patients in the 20102012 cohort were diagnosed in the first 4 months after T1D diagnosis as opposed to none of the patients in the 2007-2009 cohort.

screen, two of whom (14.2\%) had a positive biopsy for celiac disease. One of those two also had an increased incidence of hypoglycemic episodes and weight loss. Interestingly, he was diagnosed with biopsy-proven celiac disease five years after the diagnosis of T1DM was made (Figure 1).

As previously mentioned, for the purposes of statistical analysis, when making the timing and CD prevalence comparisons, only patients who were newly diagnosed with T1D were selected and described in the following paragraph. In the 2007-2009 cohort, out of 199 newly diagnosed T1D patients, 185 were screened for CD. Of those 185 screened,
14 were serologically positive, and seven had biopsy proven celiac disease. In the 2010-2012 cohort, of those 235 patients newly diagnosed with T1D, 213 were screened for CD. Of the 213 screened patients, 18 were serologically positive, and ten had biopsy proven CD. Of those 185 patients in the 2007-2009 cohort, $45 \%$ (83 patients) were screened in the first half year, contrasted with almost 70\% (149 patients) of those 213 who were screened in the 2010-2012 cohort (Figure 2). About 20\% $(2 / 10)$ of biopsy proven CD patients in the second cohort were diagnosed in the first 4 months after T1D diagnosis as opposed to none of the patients in the 2007-2009 cohort (Figure 3). It is important to note, however, the entire first cohort was followed for about 11 years, and some CD patients were first detected as long as 10 years after diagnosis of T1D was made.

\section{Results 2010-2012 cohort}

Of the total 250 patients seen in our center from 2010-2012, 229 (91.6\%) were screened for celiac disease. Two patients (0.8\%) were diagnosed with celiac disease prior to the diagnosis of T1D. Three of 229 (1.3\%) screened were diagnosed with IgA deficiency that renders standard $C D$ screening unreliable. One of these 3 patients had a negative biopsy result. The other 2 were not symptomatic, were not referred to gastroenterology, nor biopsied. Of the total of 229 screened, 16 (7\%) were serologically positive on initial testing (Figure 4).

Seven of eight who were biopsied, were found to have biopsy positive CD. Again, only a minority $(28.6 \%)$, two of 7 $\mathrm{CD}$ patients were symptomatic. In the symptomatic group only one of the two patients had a gastrointestinal complaint (abdominal pain), and the other one had a hypoglycemic seizure. Out of those 16 patients who were serologically positive on the first screen, fifty percent (8 patients) did not have a small intestinal biopsy at first. Three of those 8 were seen by a gastroenterologist and small intestinal biopsies were recommended, however the parents refused the procedure. One of those 8 had a repeat strongly positive screen and subsequently was found to have biopsy proven $C D$. The other four patients had weakly positive tissue transglutaminase titers ranging between $25-28 \mathrm{U} / \mathrm{ml}$. Antigliadin IgA and antiendomysial IgA titers were negative in all four patients. Thus, follow-up antibody titers were recommended in 3-6 months. In addition, HLA testing was performed in one of these 4 patients and was negative, making the diagnosis of celiac disease unlikely. In summary, seven of those eight patients serologically positive on their first screen who were not biopsied, subsequently had their anti-TTG antibody levels normalize, four of whom had known negative antiendomysial $\lg \mathrm{A}$ titers (Figure 4).

\section{Statistical analysis}

Of those 10 biopsy-proven CD patients in the second cohort, 7 (70\%) were diagnosed with CD within the first 1 year (Figure 3 ). The prevalence of CD in the 2010-2012 cohort was 4.7\% (10 of 213 screened), slightly higher, but not statistically different 


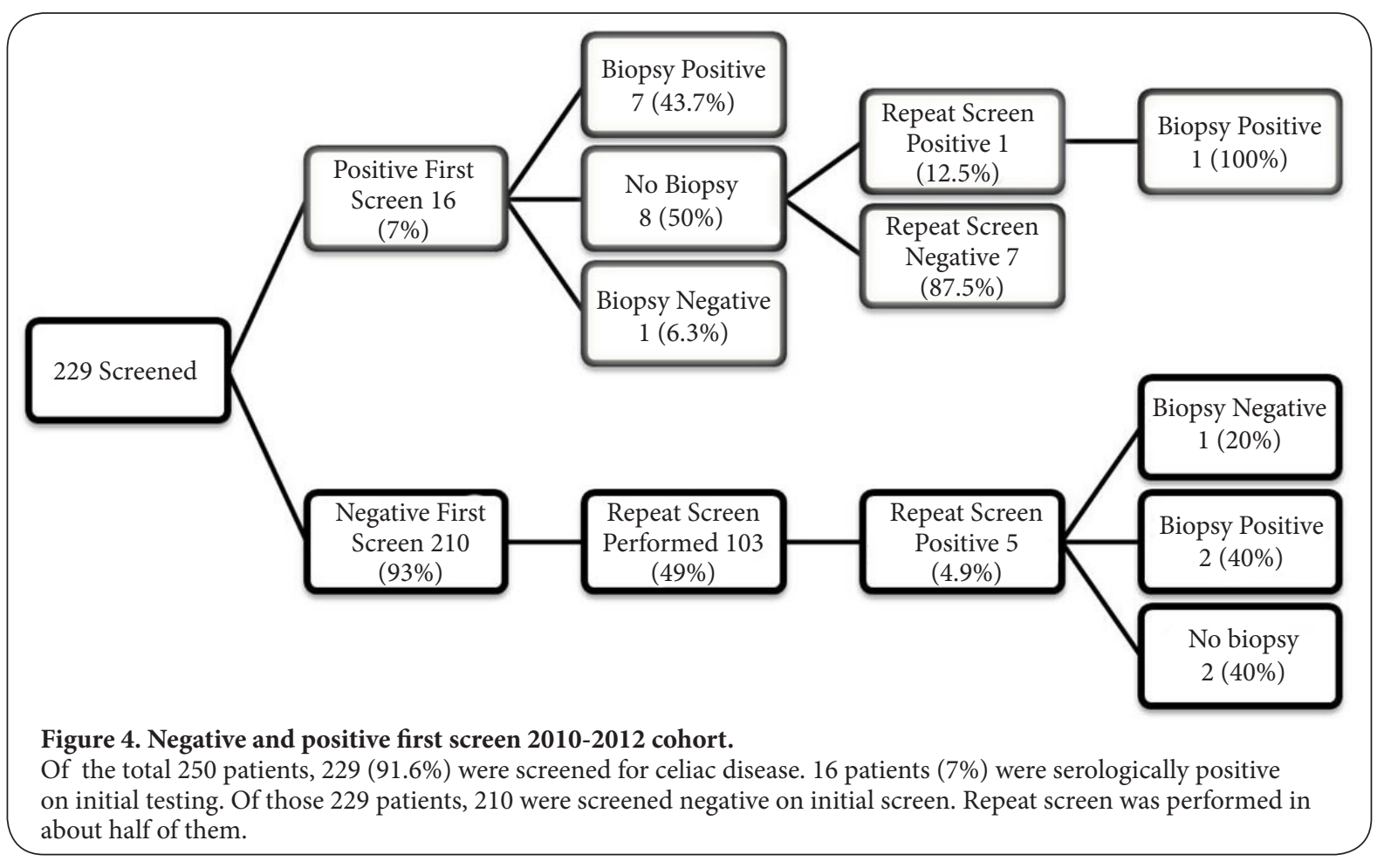

from the prevalence found in the 2007-2009 cohort of 3.8\% (7 of 185 screened), using the two sample proportion test $\mathrm{x}^{2}=0.2, \mathrm{df}=1, \mathrm{p}$ value $=0.65$.

Using the Kaplan-Meier (KM) estimator the mean time to screening in the first cohort was $0.87 \pm 0.06$ years compared to $0.54 \pm 0.03$ years in the second cohort $\left(X^{2}=19.4, D F=1, p\right.$-value $<0.0001$ ) [17]. The time to screening in 2007-2009 cohort (out of those newly diagnosed patients only) was significantly longer than in the 2010-2012 cohort. Of the patients who tested positive for celiac disease, the time to diagnosis of CD was not statistically different between the two cohorts, $1.36 \pm 0.32$ years in the first part of our study compared to $1.13 \pm 0.13$ years in the second part $\left(X^{2}=0.49, D F=1, p\right.$-value $\left.<0.48\right)$.

\section{Discussion}

CD occurs more commonly in children with T1D than in general population [1]. Our study has confirmed a high prevalence of biopsy-proven $\mathrm{CD}$ in this high risk population $(4.7 \%$ in our second cohort and $3.8 \%$ in the first one), which is about 4 times higher than the estimated prevalence in the general population [3]. Only a minority ( 1 of 7 patients) in our first cohort recognized Gl symptoms before a definitive diagnosis was made. However, 3 of those 7 patients were 4 years old or younger, and such young patients may not be able to articulate symptoms. Most patients (70\%) in the second cohort were also asymptomatic; one patient had complaints of abdominal pain and the others had an increased incidence of hypoglycemic episodes. Similar findings were reported by Sumnik et al., [18] and Barera et al [19]. We believe that symptoms should not be a prerequisite for $C D$ screening in this high risk population. Our data bolster the ADA's, NASPGHAN's, ISPAD's and ESPGHAN's positions that pediatric endocrinologists should screen all patients with T1D for celiac disease soon after diabetes is diagnosed, and further propose regular interval re-screening for those who test negative initially. However, these findings challenge the currently published $C D$ screening guidelines from AGA and ACG that do not reflect the need to screen all asymptomatic T1D patients.

With institution of the screening guidelines at our institution we were able to demonstrate improvement in earlier screening of $C D$ in this high risk population. In addition in our second cohort, after the new screening guidelines were implemented, $70 \%$ (7 out of 10) biopsy proven CD patients were diagnosed within the first year. These findings agree with previous reports in both the timing \& prevalence of $C D$. The majority of cases (85\%) are diagnosed $2-5$ years following the T1DM clinical onset [5]. The mean time to diagnosis of CD even in our second cohort, after implementing of the new screening guidelines was about 1 year. However, in a small minority of our patients ( $0.8 \%)$ in the second cohort, CD was a preexisting diagnosis prior to the diagnosis of T1D, which is consistent with previously published data [19]. Furthermore, in $87.5 \%(7 / 8)$ of patients in our second cohort who had weakly positive TTG IgA titers $(<30 \mathrm{U} / \mathrm{ml})$, antibodies normalized on subsequent screening, even as they continued to consume gluten. These findings are consistent with previous reports of normalization of celiac titers in some T1D patients not on gluten-free diet [20]. 
There are several limitations to our study. In both cohorts, a minority of patients, $16 \%$ of those 25 with a positive first screen in the 2007-2009 cohort (Figure 1), and 6.3\% of those 16 with a positive first screen in the second cohort (Figure 4), were found to have biopsies negative for CD. We continued to follow them periodically after the first 2-3 years of T1D diagnosis. However, to date there are no controlled trials to guide recommendations for asymptomatic children with elevated anti-TTG titers and normal small bowel biopsies. Likewise, the optimal frequency of repeat serologic testing of these patients or those with negative celiac titers is lacking.

In this regard, the lack of a clearly defined, detailed algorithm for screening which could be uniformly applied was a significant disadvantage of our study. The appearance of just such an algorithm for asymptomatic individuals at higher risk for CD by the ESPGHAN [15] towards the end of our study could obviate many of these problems. There are at least two difficulties in applying this algorithm to our US population. First is the reasonable recommendation to start screening with HLA DQ2/DQ8 genetic testing. This could eliminate that proportion of the population who do not need celiac antibody screening now or in the future. However, HLA DQ2/DQ8 genotyping is relatively expensive at two to three times the cost of antibody titers, may not be covered by insurance for this purpose, and is not available in many commercial laboratories. The second difficulty is the widespread variability in the performance of the various celiac antibodies in multiple commercial laboratories. This makes the decision to obtain confirmatory EMA testing specifically for a positive but less than three times the upper limit of normal TTG-A test as in the ESPGHAN recommendations problematic [15].

\section{Conclusions}

In conclusion, based on the data collected in our center and building on other published guidelines, we propose screening patients with T1D at diagnosis according to the age-based algorithm below.

Obtain HLA DQ testing if feasible given insurance and laboratory constraints. For those that are DQ2/DQ8 positive or who have not undergone HLA testing:

For patients $>2$ years old, a tissue transglutaminase IgA (TTGA) level and serum IgA level;

- If weakly positive TTG-A, proceed with Antiendomysial IgA titer (EMA),

- If EMA is positive -- refer to Gastroenterology (Gl)

- If EMA negative, repeat titers in 6 months

- If strongly positive TTG-A, refer to GI

- If IgA deficiency-refer to GI

- If negative TTG-A and serum IgA level normal-- repeat

TTG-A yearly for five years and then every 2-3 years thereafter

For patients $<2$ yrs. old, a TTG-A level, a deamidated antigliadin $\lg \mathrm{A}$, and quantitative serum IgA level.

- If negative TTG-A and positive deamidated antigliadin IgA -- refer to Gl
- If positive TTG-A and positive deamidated antigliadin IgA -- refer to Gl

- If weakly positive TTG-A and positive/negative deamidated antigliadin IgA—refer to $\mathrm{Gl}$

- If IgA deficiency - refer to GI

- If TTG-A and deamidated antigliadin IgA both negative, and serum IgA level normal, repeat TTG-A yearly for five years and then every 2-3 years thereafter.

Our research suggests that patients with T1D should be screened regardless of overt gastroenterological symptoms of $C D$. This will allow early dietary intervention and reduce morbidities in this high-risk population. Since manifestations of celiac disease are often subtle, early screening is vital.

\section{Competing interests}

The authors declare that they have no competing interests.

Authors' contributions

\begin{tabular}{|l|c|c|c|c|c|c|}
\hline Authors' contributions & LG & MJP & DEC & GRF & PMK & PWS \\
\hline Research concept and design & $\checkmark$ & -- & -- & -- & -- & $\checkmark$ \\
\hline Collection and/or assembly of data & $\checkmark$ & $\checkmark$ & $\checkmark$ & $\checkmark$ & $\checkmark$ & $\checkmark$ \\
\hline Data analysis and interpretation & $\checkmark$ & $\checkmark$ & -- & -- & -- & $\checkmark$ \\
\hline Writing the article & $\checkmark$ & $\checkmark$ & -- & -- & -- & $\checkmark$ \\
\hline Critical revision of the article & -- & $\checkmark$ & -- & -- & -- & $\checkmark$ \\
\hline Final approval of article & $\checkmark$ & $\checkmark$ & $\checkmark$ & $\checkmark$ & $\checkmark$ & $\checkmark$ \\
\hline Statistical analysis & $\checkmark$ & -- & -- & -- & -- & - \\
\hline
\end{tabular}

Acknowledgement

The authors thank Isaac J. Gabriel and Rose Calixte, for their help with statistical analysis and figure formatting for the manuscript.

\section{Publication history}

EIC: Geoffrey Burnstock, University College London, UK. Received: 26-Oct-2014 Final Revised: 10-Jan-2015

Accepted: 22-Jan-2015 Published: 27-Jan-2015

\section{References}

1. Plenge RM. Shared genetic risk factors for type $\mathbf{1}$ diabetes and celiac disease. N Engl J Med. 2008; 359:2837-8. I Article I PubMed

2. Volta $U$, Tovoli $F$ and Caio G. Clinical and immunological features of celiac disease in patients with Type 1 diabetes mellitus. Expert Rev Gastroenterol Hepatol. 2011; 5:479-87. | Article I PubMed

3. Guandalini S and Assiri A. Celiac disease: a review. JAMA Pediatr. 2014; 168:272-8. | Article | PubMed

4. Hill ID, Dirks MH, Liptak GS, Colletti RB, Fasano A, Guandalini S, Hoffenberg EJ, Horvath K, Murray JA, Pivor M and Seidman EG. Guideline for the diagnosis and treatment of celiac disease in children: recommendations of the North American Society for Pediatric Gastroenterology, Hepatology and Nutrition. J Pediatr Gastroenterol Nutr. 2005; 40:1-19. | Article | PubMed

5. AGA Institute Medical Position Statement on the Diagnosis and Management of Celiac Disease. Gastroenterology. 2006; 131:1977-80. | Article | PubMed Abstract | PubMed Full Text

6. Kordonouri O, Maguire AM, Knip M, Schober E, Lorini R, Holl RW and Donaghue KC. Other complications and associated conditions with diabetes in children and adolescents. Pediatr Diabetes. 2009; 10 Suppl 12:204-10. | Article | PubMed 
7. Standards of medical care in diabetes--2012. Diabetes Care. 2012; 35 Suppl 1:S11-63. | Article | PubMed Abstract | PubMed Full Text

8. Kakleas K, Karayianni C, Critselis E, Papathanasiou A, Petrou V, Fotinou $A$ and Karavanaki $K$. The prevalence and risk factors for coeliac disease among children and adolescents with type 1 diabetes mellitus. Diabetes Res Clin Pract. 2010; 90:202-8. | Article | PubMed

9. Narula P, Porter L, Langton J, Rao V, Davies P, Cummins C, Kirk J, Barrett $T$ and Protheroe $S$. Gastrointestinal symptoms in children with type 1 diabetes screened for celiac disease. Pediatrics. 2009; 124:e489-95. | Article | PubMed

10. Mohn A, Cerruto M, lafusco D, Prisco F, Tumini S, Stoppoloni O and Chiarelli F. Celiac disease in children and adolescents with type I diabetes: importance of hypoglycemia. J Pediatr Gastroenterol Nutr. 2001; 32:37-40. | Article | PubMed

11. Hansen D, Brock-Jacobsen B, Lund E, Bjorn C, Hansen LP, Nielsen C, Fenger C, Lillevang ST and Husby S. Clinical benefit of a gluten-free diet in type 1 diabetic children with screening-detected celiac disease: a population-based screening study with 2 years' follow-up. Diabetes Care. 2006; 29:2452-6. | Article | PubMed

12. Rubio-Tapia A, Hill ID, Kelly CP, Calderwood AH and Murray JA. ACG clinical guidelines: diagnosis and management of celiac disease. Am J Gastroenterol. 2013; 108:656-76. | Article | PubMed Abstract | PubMed Full Text

13. Olen O, Gudjonsdottir AH, Browaldh L, Hessami M, Elvin K, Liedberg AS, Neovius $M$ and Grahnquist L. Antibodies against deamidated gliadin peptides and tissue transglutaminase for diagnosis of pediatric celiac disease. J Pediatr Gastroenterol Nutr. 2012; 55:695-700. | Article | PubMed

14. Brusca I, Carroccio A, Tonutti E, Villalta D, Tozzoli R, Barrale M, Sarullo FM, Mansueto P, Chiusa SM, lacono G and Bizzaro N. The old and new tests for celiac disease: which is the best test combination to diagnose celiac disease in pediatric patients? Clin Chem Lab Med. 2012; 50:111-7. | Article | PubMed

15. Husby S, Koletzko S, Korponay-Szabo IR, Mearin ML, Phillips A, Shamir R, Troncone R, Giersiepen K, Branski D, Catassi C, Lelgeman M, Maki M, Ribes-Koninckx C, Ventura A and Zimmer KP. European Society for Pediatric Gastroenterology, Hepatology, and Nutrition guidelines for the diagnosis of coeliac disease. J Pediatr Gastroenterol Nutr. 2012; 54:136-60. | Article | PubMed

16. Marsh MN. Gluten, major histocompatibility complex, and the small intestine. A molecular and immunobiologic approach to the spectrum of gluten sensitivity ('celiac sprue'). Gastroenterology. 1992; 102:330-54. I PubMed

17. Kaplan EL MP. Nonparametric estimation from incomplete observations. Journal of the American statistical association. 1958; 53:457-481.

18. Sumnik Z, Cinek O, Bratanic N, Lebl J, Rozsai B, Limbert C, Paskova M and Schober $E$. Thyroid autoimmunity in children with coexisting type 1 diabetes mellitus and celiac disease: a multicenter study. J Pediatr Endocrinol Metab. 2006; 19:517-22. | Article | PubMed

19. Barera G, Bonfanti R, Viscardi M, Bazzigaluppi E, Calori G, Meschi F, Bianchi $C$ and Chiumello $G$. Occurrence of celiac disease after onset of type 1 diabetes: a 6-year prospective longitudinal study. Pediatrics. 2002; 109:833-8. | Article | PubMed

20. Waisbourd-Zinman O, Hojsak I, Rosenbach Y, Mozer-Glassberg Y, Shalitin S, Phillip M and Shamir R. Spontaneous normalization of anti-tissue transglutaminase antibody levels is common in children with type 1 diabetes mellitus. Dig Dis Sci. 2012; 57:1314-20. | Article | PubMed

\section{Citation:}

Gabriel L, Pettei MJ, Carey DE, Frank GR, Kreitzer PM and Speiser PW. Pediatric endocrinologists' practices in screening for celiac disease in patients with type 1 diabetes. J Diab Res Clin Met. 2015; 4:1. http://dx.doi.org/10.7243/2050-0866-4-1 